\title{
Laser Treatment of Medial Epicanthoplasty Scars
}

\author{
Sang Ju Lee ${ }^{1}$ \\ Won Soon Chung ${ }^{1}$ \\ Young Koo Kim ${ }^{1}$ \\ Eujin $\mathrm{Cho}^{2}$
}

${ }^{1}$ Yonsei Star Skin \& Laser Clinic, Seoul, Korea
${ }^{2}$ Department of Dermatology, Graduate School of
Medical Science and Engineering, KAIST,
Daejeon, Korea

Received December 9, 2016

Revised December 18, 2016

Accepted December 18, 2016

\section{Correspondence}

Eujin Cho

Department of Dermatology, Graduate School of Medical Science and Engineering, KAIST, 291

Daehak-ro, Yuseong-gu, Daejeon 34141, Korea

Tel.: +82-42-350-5170

Fax: +82-42-350-0505

E-mail: eujinchodgmail.com

(C) Korean Society for Laser Medicine and Surgery

(c) This is an open access article distributed under the terms of the Creative Commons Attribution NonCommercial License (http://creativecommons.org/ licenses/by-nc/4.0) which permits unrestricted noncommercial use, distribution, and reproduction in any medium, provided the original work is properly cited.
Medial epicanthoplasty is a commonly performed aesthetic surgery, in addition to a double-eyelid surgery. Epicanthoplasty, compared with double-eyelid surgery, may cause scarring comparably in numbers and seriousness. Various treatments have been attempted; however, to date, there have not been any reported methods better than revision surgeries. Hence, herein, we present successful cases using laser treatment after scarring due to medial epicanthoplasty.

\section{Key words}

Epicanthoplasty; Laser and light sources; Surgery 


\section{INTRODUCTION}

Medial epicanthoplasty is a commonly performed aesthetic surgery along with double-eyelid surgery. ${ }^{1}$ Epicanthoplasty causes scarring that is not less than in numbers and seriousness compared to double-eyelid surgery. Various treatments have been attempted; ${ }^{2}$ however, no significantly better method has been reported other than revision surgery. Hence, herein, we present cases successfully treated with laser after scarring due to medial epicanthoplasty.

\section{CASE REPORT}

A 20-year-old woman presented with asymptomatic, linear scarring on both inner canthi of the eyelids that had developed 2 years ago. The patient was otherwise healthy
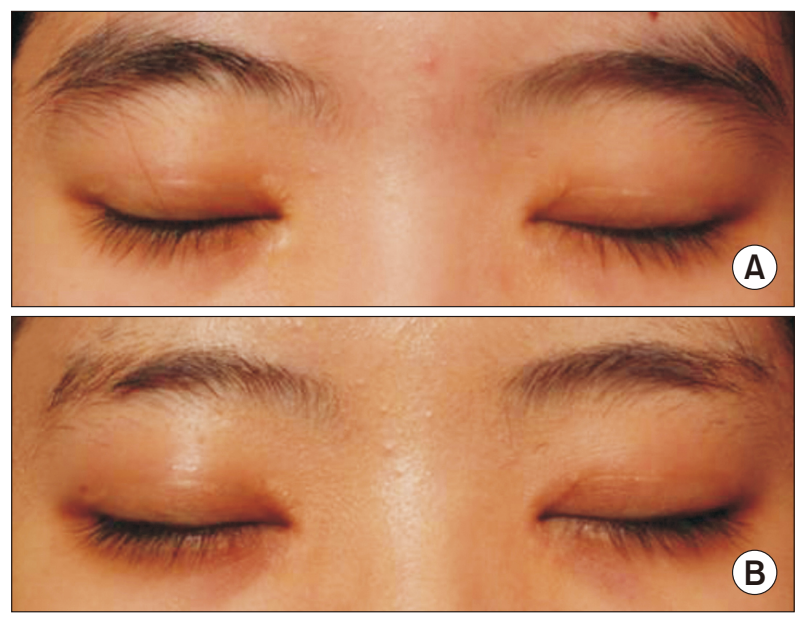

Fig. 1. Linear white depressed scar on the inner canthus of the upper eyelid, (A) before and (B) two months after two sessions of laser treatment. and had no history of trauma other than her past history of medial epicanthoplasty. Physical examination revealed linear, white, depressed scar on the inner canthi of the upper eyelids (Fig. 1A). The second patient, a 26-year-old woman, presented with asymptomatic, linear scarring on her right lower eyelid that had developed 6 months ago. The patient was otherwise healthy and had no other history of trauma except for the medial epicanthoplasty. Physical examination revealed linear, elevated scar on the right lower eyelid (Fig. 2A). For the patients, a 10,600$\mathrm{nm} \mathrm{CO} 2$ laser (Ultrapulse Encore; Lumenis, Santa Clara, CA, USAl was used. The Deep FX mode of $20 \mathrm{~mJ}$, density $1(5 \%)$ was performed. Topical application of EMLA cream (AstraZeneca AB, Södertälje, Sweden) was used for local anesthesia one hour prior to the procedure. Two to three sessions of ablative fractional $\mathrm{CO}_{2}$ laser and Starlux 1540$\mathrm{nm}$ nonablative fractional laser (Palomar, Westford, MA, USA) at 30-50 mJ using a $10 \mathrm{~mm}$ spot in intervals of four to eight weeks were performed. A combination of ablative and nonblative fractional laser was used to maximize the efficacy and lower potential side effects. The scars were softened, flattened with improved texture in both patients at the two months and four months follow-up, respectively (Figs. 1B and 2B). Post-therapy transient erythema and crusting spontaneously resolved along with clinical improvements.

\section{DISCUSSION}

Epicanthus is a vertical fold of skin on either side of the nose from the medial canthus. In Asians, it presents as a normal characteristic. However, because it covers the inner canthus, in some cases it may seem like telecanthus. As a result, medial epicanthoplasty has become very common for aesthetic purposes. Furthermore, if medial epicanthoplasty is performed with double-eyelid surgery,
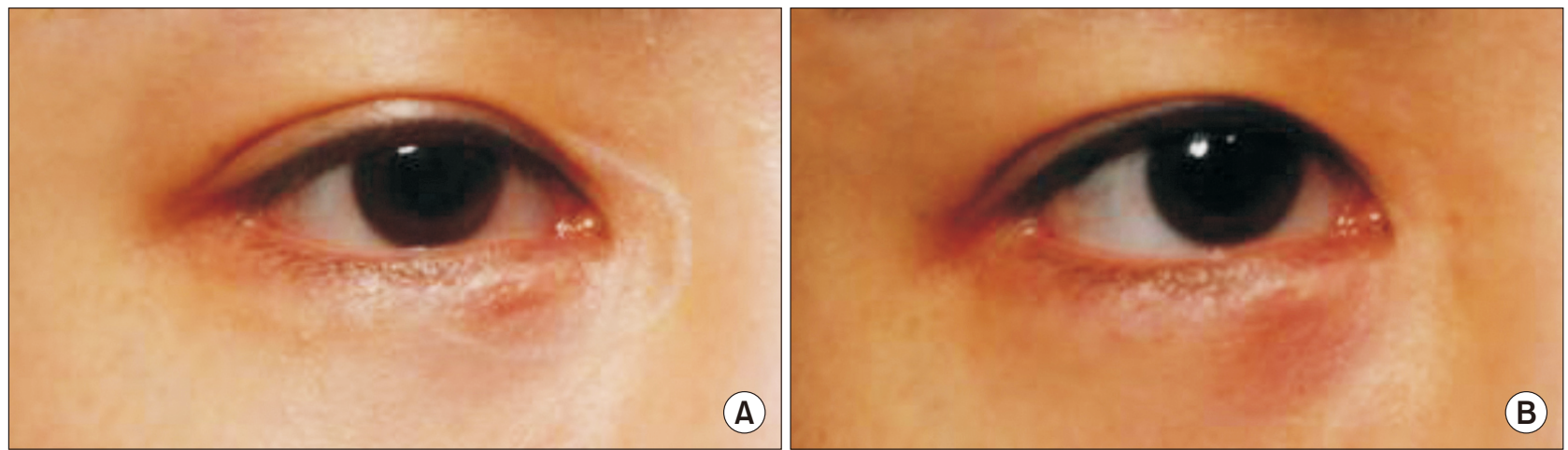

Fig. 2. Linear elevated scar on the right lower eyelid, (A) before and (B) four months after three sessions of laser treatment. 
the double eyelids may be able to have more of a natural appearance. ${ }^{1}$ Therefore, medial epicanthoplasty along with double-eyelid surgery are likely to be operated simultaneously to achieve larger and clearer eyes. In spite of the anticipation of having better aesthetic results with the combined surgery, scarring is a major concern. ${ }^{2}$ The possibility of leaving scar with medial epicanthoplasty outweighs that of double-eyelid surgery. Various modalities have been attempted to reduce the chance of scarring. ${ }^{1,2}$ However, the outcomes of different treatment methods have not been as satisfactory as revision surgery until now. Fractional lasers are well-known for its use in treating and improving the texture and appearance of scars. ${ }^{3,4}$ Fractional lasers may have potential advantages in such cases because of their ability to penetrate into the deep dermis. ${ }^{3} \mathrm{CO}_{2}$ lasers used in a fractional pattern ablate abnormal scars and allow formation of neocollagen and rapid reepithelialization of the skin surface. ${ }^{4-6}$ With no other treatments as successful as revision surgery in cases of scarring after medial epicanthoplasty, fractional laser treatment may be a safe and effective treatment modality with almost no downtime.

We report two cases of medial epicanthoplasty scarring successfully treated with fractional laser. Therefore, in our experience, fractional lasers can be an effective ther- apeutic option for the treatment of medial epicanthoplasty scars.

\section{REFERENCES}

1. Park DH, Park SU, Ji SY, Baik BS. Combined epicanthoplasty and blepharoptosis correction in Asian patients. Plast Reconstr Surg 2013;132:510e-9e.

2. Park DH, Park SU, Lee BK, Lee YB, Do ER, Han DG, et al. Medial epicanthoplasty without a vertical scar. Ann Plast Surg 2014;73:8-11.

3. Cho SB, Lee SJ, Chung WS, Kang JM, Kim YK. Treatment of burn scar using a carbon dioxide fractional laser. J Drugs Dermatol 2010;9:173-5.

4. Cho SB, Lee SJ, Kang JM, Kim YK, Kim TY, Kim DH. The treatment of burn scar-induced contracture with the pinhole method and collagen induction therapy: a case report. J Eur Acad Dermatol Venereol 2008;22:513-4.

5. Whang SW, Lee KY, Cho SB, Lee SJ, Kang JM, Kim YK, et al. Burn scars treated by pinhole method using a carbon dioxide laser. J Dermatol 2006;33:869-72.

6. Lee SJ, Cho S, Kim YK, Cho SB. Scar revision by the pinhole method using a 10,600-nm carbon dioxide laser. J Cosmet Laser Ther 2013;15:37-8. 